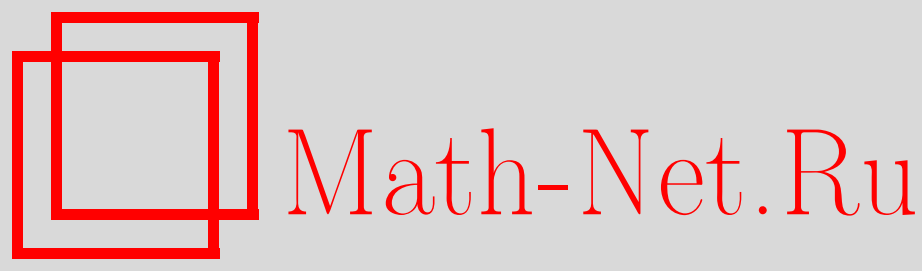

В. М. Журавлев, Метод обобщенных подстановок Коула-Хопфа и новые примеры линеаризуемых нелинейных эволюционных уравнений, ТМФ, 2009, том 158, номер 1, 58-71

DOI: https://doi.org/10.4213/tmf6299

Использование Общероссийского математического портала Math-Net.Ru подразумевает, что вы прочитали и согласны с пользовательским соглашением http://www . mathnet.ru/rus/agreement

Параметры загрузки:

IP : 3.80 .253 .173

26 апреля 2023 г., 14:06:33

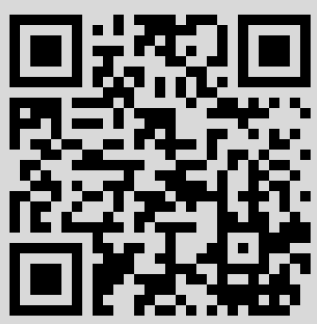




\title{
МЕТОД ОБОБЩЕННЫХ ПОДСТАНОВОК КОУЛА-ХОПФА И НОВЫЕ ПРИМЕРЫ ЛИНЕАРИЗУЕМЫХ НЕЛИНЕЙНЫХ ЭВОЛЮЦИОННЫХ УРАВНЕНИЙ
}

\begin{abstract}
Предложен новый подход к конструированию нелинейных эволюционных уравнений в матричной форме, интегрируемых с помощью подстановок, аналогичных подстановке Коула-Хопфа, линеаризующей уравнение Бюргерса. С помощью нового подхода найдены новые интегрируемые нелинейные эволюционные уравнения и их иерархии.
\end{abstract}

Ключевые слова: точно интегрируемые нелинейные уравнения, уравнения типа Бюргерса, подстановки Коула-Хопфа.

\section{1. ВВЕДЕНИЕ}

До появления развитого метода обратной задачи (МО3), связанного с представлением нелинейных волновых уравнений в форме условия коммутативности двух или более дифференциальных операторов, одним из наиболее важных результатов в теории нелинейных уравнений математической физики был факт интегрируемости уравнения Бюргерса с помощью линеаризующей подстановки Коула-Хопфа [1], [2]. Вид подстановки Коула-Хопфа сыграл определенную роль в создании МОЗ [2]. Например, подстановка Миуры [3], в некотором смысле аналогичная подстановке Коула-Хопфа, преобразующая уравнение Кортевега-де Фриза (КдФ) в модифицированное уравнение Кортевега-де Фриза (мКдФ), позволила получить бесконечный набор нетривиальных законов сохранения для обоих этих уравнений и доказать их полную интегрируемость.

В дальнейшем метод подстановок типа Коула-Хопфа был существенно расширен. В работе [4] этот метод был распространен на класс эволюционных уравнений вида $u_{t}=u_{n}+f\left(u, u_{1}, \ldots, u_{n-1}\right)$, где $u=u(x, t)$ - функция двух переменных, $u_{n}=\partial^{n} u / \partial x^{n}$, и составлен полный список уравнений, которые линеаризуются с помощью подстановки Коула-Хопфа. Такие уравнения получили название уравнений типа Бюргерса. В дальнейшем, при развитии симметрийного подхода в теории интегрируемости нелинейных уравнений, уравнения типа Бюргерса обычно приводились

*Ульяновский государственный университет, Ульяновск, Россия. E-mail: zhvictorm@gmail.com 
в качестве дополнения к спискам интегрируемых уравнений из-за наличия у них бесконечных серий законов сохранения [5]-[10]. С помощью симметрийного подхода (инварианты Лапласа), в работах [11], [12] был рассмотрен метод нахождения уравнений, которые с помощью дифференциальных подстановок могут быть преобразованы в уравнения аналогичного типа. Матричное обобщение метода подстановки Коула-Хопфа, называемое $C$-интегрируемостью, было предложено в работе [13]. Связь этого метода с другими методами интегрирования нелинейных дифференциальных уравнений в частных производных была рассмотрена в работе [14], где содержится достаточно полная библиография по этой теме (см. также работу [15]). Кроме того, методы, аналогичные подстановкам Коула-Хопфа в виде преобразований Беклунда и методов факторизации в рамках симметрийного подхода, использовались в задаче классификации интегрируемых нелинейных уравнений [5]-[10]. В этих работах была проведена классификация всех нелинейных уравнений, которые обладают бесконечной серией законов сохранения. Однако при таком симметрийном подходе нет прямой возможности сразу в явном виде указать метод построения всех точных решений изучаемых уравнений после доказательства их интегрируемости. Эта проблема обычно решалась в тех случаях, когда для уравнения явно указывалось представление Лакса-Захарова-Шабата (ЛЗШ) или преобразование, сводящее его к уравнению, имеющему представление ЛЗШ. Для таких уравнений можно сразу в явном виде указать их точные солитонные или периодические решения (см., например, [16]-[19] и библиографию в них). Аналогичным свойством обладают уравнения, которые линеаризуются с помощью подстановок типа Коула-Хопфа. Для МОЗ построены алгоритмы вычисления представлений типа ЛЗШ и истинных представлений ЛЗШ даже для многомерных уравнений (см. [18], [19] и библиографию в них). Аналогичный универсальный алгоритм для метода подстановок типа Коула-Хопфа был найден в работах [20]-[22], где с помощью результатов работы [23] получен новый метод построения интегрируемых уравнений типа Бюргерса и их точных решений, основанный на обобщенных линеаризующих подстановках типа Коула-Хопфа, который фактически дает общий алгоритм построения таких уравнений. Идея метода состоит в использовании замыкания совокупности линейных дифференциальных соотношений относительно некоторого набора смешанных производных функции, удовлетворяющей уравнению теплопроводности и уравнению переноса изолиний этой функции [21], [23].

В настоящей работе показано, что результаты работ [21], [22] можно обобщить и применить к построению широкого класса нелинейных уравнений и их систем, линеаризуемых с помощью подстановок типа Коула-Хопфа. Перечислены все возможные уравнения типа Бюргерса в размерности $1+1$, в том числе и матричные. Приведен ряд примеров использования этого метода для нахождения конкретных уравнений, в том числе уравнений динамики сжимаемой жидкости.

\section{2. ОБОБЩЕННЫЙ МЕТОД ПОДСТАНОВОК КОУЛА-ХОПФА}

Как отмечалось в разделе 1 , подход, основанный на матричном обобщении подстановок Коула-Хопфа, называется $C$-интегрируемостью [13], [14]. Приведенные ниже результаты дают общий алгоритм построения матричных уравнений, интегрируемых с помощью матричного аналога подстановок типа Коула-Хопфа. 
Следуя работам [21], [22], рассмотрим следующую пару матричных уравнений относительно матричной функции $\widehat{T}$ :

$$
\begin{gathered}
\widehat{T}_{t}-\widehat{D} \widehat{T}_{x x}+\widehat{W} \widehat{T}_{x}=0, \\
\widehat{T}_{t}+\widehat{V} \widehat{T}_{x}=0,
\end{gathered}
$$

где $\widehat{T}, \widehat{W}, \widehat{V}, \widehat{D}$ - квадратные $(M \times M)$-матрицы, элементы которых являются функциями $x$ и $t$. Вычисляя совокупность производных согласно [22] для скалярного случая, получаем систему соотношений для производных в следующем виде:

$$
\begin{aligned}
& \widehat{T}_{t t}-\widehat{D} \widehat{T}_{x x t}+\widehat{W}_{t} \widehat{T}_{x}+\widehat{W} \widehat{T}_{x t}-\widehat{D}_{t} \widehat{T}_{x x}=0, \\
& \widehat{T}_{t t}+\widehat{V}_{t} \widehat{T}_{x}+\widehat{V} \widehat{T}_{t x}=0, \\
& \widehat{T}_{x t}-\widehat{D} \widehat{T}_{x x x}+\widehat{W}_{x} \widehat{T}_{x}+\widehat{W} \widehat{T}_{x x}-\widehat{D}_{x} \widehat{T}_{x x}=0, \\
& \widehat{T}_{x t}+\widehat{V}_{x} \widehat{T}_{x}+\widehat{V} \widehat{T}_{x x}=0 \\
& \widehat{T}_{t x x}+\widehat{V}_{x x} \widehat{T}_{x}+2 \widehat{V}_{x} \widehat{T}_{x x}+\widehat{V} \widehat{T}_{x x x}=0 .
\end{aligned}
$$

Исключая последовательно из этих уравнений все высшие производные матрицы $\widehat{T}$, получаем систему базовых соотношений:

$$
\begin{aligned}
& \widehat{T}_{t}=-\widehat{V} \widehat{T}_{x}, \quad \widehat{T}_{x x}=-\widehat{U} \widehat{T}_{x}, \quad \widehat{T}_{x t}=-\widehat{Q} \widehat{T}_{x}, \\
& \widehat{T}_{t t}=-\left[V_{t}-\widehat{V} \widehat{Q}\right] \widehat{T}_{x}, \\
& \widehat{T}_{x x x}=\left[-\widehat{U}_{x}+\widehat{U}^{2}\right] \widehat{T}_{x}, \\
& \widehat{T}_{x x t}=\left[-\widehat{U}_{t}+\widehat{U} \widehat{Q}\right] \widehat{T}_{x}=\left[-\widehat{V}_{x x}+\widehat{Q} \widehat{U}+\widehat{V}_{x} \widehat{U}+\widehat{V} \widehat{U}_{x}\right] \widehat{T}_{x},
\end{aligned}
$$

где $\widehat{U}=\widehat{D}^{-1}(\widehat{V}-\widehat{W}), \widehat{Q}=\widehat{V}_{x}-\widehat{V} \widehat{U}$. Сравнивая два выражения, полученных для $\widehat{T}_{x x t}$, приходим к следующему уравнению совместности этой системы:

$$
\widehat{U}_{t}+[\widehat{Q}, \widehat{U}]+\frac{\partial}{\partial x}(\widehat{V} \widehat{U})-\widehat{V}_{x x}=0
$$

или

$$
\widehat{U}_{t}+[\widehat{Q}, \widehat{U}]-\frac{\partial}{\partial x} \widehat{Q}=0
$$

где $[\widehat{Q}, \widehat{U}]=\widehat{Q} \widehat{U}-\widehat{U} \widehat{Q}$ - коммутатор матриц. Уравнение (6), как нетрудно видеть, является уравнением нулевой кривизны Захарова-Шабата [16] для двух линейных матричных операторов первого порядка:

$$
\Psi_{x}=\widehat{U} \Psi, \quad \Psi_{t}=\widehat{Q} \Psi
$$

где $\Psi=\widehat{T}_{x}$. Это уравнение является центральным в теории МОЗ. Однако, в отличие от МО3, при таком подходе не требуется наличия спектрального параметра для построения решений уравнений, соответствующих (6). Точные решения получаются из базовых соотношений (4), а дополнительная связь возникает из дополнительного условия для матричной функции $\widehat{T}$. 
Таким образом, полученный результат сводится к тому, что для любой матричной функции $\widehat{T}(x, t)$, имеющей все смешанные производные до третьего порядка включительно в заданной области переменных $x, t$, матричные функции $\widehat{U}(x, t)$ и $\widehat{V}(x, t)$, связанные с $\widehat{T}(x, t)$ соотношениями (4), удовлетворяют структурному уравнению (6).

Высшие производные матричной функции $T$ связаны с производной $\widehat{T}_{x}$ соотношениями следующего общего вида:

$$
\widehat{T}^{[n, k]}=\hat{A}^{n k}[\widehat{V}, \widehat{U}] \widehat{T}_{x},
$$

где матричные функции $\hat{A}^{n k}[\widehat{V}, \widehat{U}]$ являются дифференциальными полиномами от матриц $\widehat{V}, \widehat{U}$ и их производных. Дифференциальные полиномы $\hat{A}^{n k}[\widehat{V}, \widehat{U}]$ удовлетворяют следующим рекуррентным соотношениям:

$$
\begin{gathered}
\hat{A}^{n+1 k}=\hat{A}_{x}^{n k}+\hat{A}^{n k} \hat{A}^{20}, \\
\hat{A}^{n k+1}=\hat{A}_{t}^{n k}+\hat{A}^{n k} \hat{A}^{11}, \\
\hat{A}^{20}=-\widehat{U}, \quad \hat{A}^{11}=-\widehat{Q}, \quad n, k=0,1,2 \ldots,
\end{gathered}
$$

которые получаются из расширенной системы базовых соотношений (4), (7) дифференцированием по $x$ и $t$ до любого конечного порядка $[k, n]$ (при естественном предположении существования производных соответствующего порядка матричной функции $\widehat{T})$.

Пусть существуют матричные функции $\widehat{C}_{k n}(x, t)$ такие, что между функциями $\widehat{U}$ и $\widehat{V}$ имеется следующая связь:

$$
\sum_{k=0}^{M} \sum_{n=1}^{N} \widehat{C}_{k n}(x, t) \hat{A}^{k n}[\widehat{V}, \widehat{U}]=0 .
$$

Тогда функция $\widehat{T}$ удовлетворяет линейному дифференциальному уравнению

$$
\sum_{k=0}^{M} \sum_{n=1}^{N} \widehat{C}_{k n} \widehat{T}^{[k, n]}=0,
$$

при этом совместное решение относительно функций $\widehat{U}, \widehat{V}$ и $\widehat{Q}$ уравнения (6) со связью (9) имеет вид

$$
\widehat{V}=-\widehat{T}_{t}\left[\widehat{T}_{x}\right]^{-1}, \quad U=-\widehat{T}_{x x}\left[\widehat{T}_{x}\right]^{-1}, \quad \widehat{Q}=-\widehat{T}_{x t}\left[\widehat{T}_{x}\right]^{-1} .
$$

Последние соотношения вместе с формулой (7) назовем обобщенными подстановками Коула-Хопфа. Этот результат является обобщением результата работы [22] и служит основой для вычисления всей совокупности уравнений типа Бюргерса. Сформулируем его в виде следующего утверждения.

УтВЕРЖДЕНИЕ. Любое нелинейное уравнение типа Бюргерса конечного порядка, линеаризуемое с помощью обобщенных подстановок Коула-Хопфа (11) и, возможно, (7), может быть представлено системой двух уравнений (9) и (5).

Справедливость данного утверждения вытекает из следующих фактов. Списки уравнений (9) и (10) имеют взаимно однозначное соответствие, определяемое соотношениями (7), (8), вид которых не зависит ни от функциональной формы $\widehat{T}$, 
ни от вида уравнений (10). Кроме того, соотношения (7) определены для любого конечного порядка производных матричной функции $\widehat{T}$. Далее, уравнение (10) представляет собой линейное уравнение общего вида произвольного конечного порядка с переменными коэффициентами и, следовательно, представляет полный список таких уравнений. По определению уравнением типа Бюргерса называется уравнение, которое сводится к некоторому линейному уравнению с помощью подстановок (11) и, возможно, (7). Следовательно, каждому уравнению типа Бюргерса соответствует хотя бы одно линейное уравнение из списка (10) в силу полноты этого списка. Поскольку каждому уравнению из списка (10) соответствует одно уравнение (9), а все обобщенные подстановки перечислены соотношениями (7) и (8), то уравнение типа Бюргерса может быть представлено как система уравнений (9) и (5).

\section{3. СКАЛЯРНЫЕ УРАВНЕНИЯ}

В частном случае при размерности матриц $M=1$ (скалярный случай) структурное уравнение (5) преобразуется в более простое уравнение

$$
U_{t}=Q_{x}
$$

При этом функции $A^{n k}[U, V]$ будут удовлетворять рекуррентным соотношениям

$$
A^{n+1 k}=A_{x}^{n k}+A^{n k} A^{11}, \quad A^{n k+1}=A_{t}^{n k}+A^{n k} A^{02}, \quad n, k=0,1,2 \ldots,
$$

с начальными условиями

$$
\begin{gathered}
A^{01}=1, \quad A^{10}=-\frac{1}{V}, \quad A^{11}=\left[\frac{V_{x}}{V}-U\right], \\
A^{20}=\frac{U}{V}, \quad A^{02}=\left[\frac{V_{t}}{V}-V_{x}+V U\right] .
\end{gathered}
$$

В частности, для производных $T^{[n, 0]}$ имеем следующие рекуррентные соотношения:

$$
T^{[n, 0]}=\frac{1}{V} G_{n}[U] T_{t}, \quad n=1,2, \ldots,
$$

где $G_{n}[U]$ - дифференциальные выражения с постоянными коэффициентами относительно функции $U$, удовлетворяющие рекуррентному соотношению

$$
G_{n+1}[U]=\frac{\partial}{\partial x} G_{n}[U]-U G_{n}[U], \quad n=1,2, \ldots,
$$

с начальным условием

$$
G_{1}[U]=-1 .
$$

Для нескольких первых рекуррентных соотношений имеем

$$
\begin{gathered}
G_{2}=U, \quad G_{3}=U_{x}-U^{2}, \quad G_{4}=U_{x x}-3 U U_{x}+U^{3}, \\
G_{5}=U_{x x x}-4 U U_{x x}-3 U_{x}^{2}+6 U^{2} U_{x}-U^{4}, \ldots .
\end{gathered}
$$

Для производных $T^{[n, 1]}$ рекуррентные соотношения имеют вид

$$
T^{[n, 1]}=H_{n}[Q] T_{t}, \quad n=1,2, \ldots,
$$


где $H_{n}[Q]$ - дифференциальные выражения с постоянными коэффициентами относительно функции

$$
Q=\frac{\partial}{\partial x} \ln V-U
$$

Функции $H_{n}[Q]$ удовлетворяют рекуррентному соотношению

$$
H_{n+1}[Q]=\frac{\partial}{\partial x} H_{n}[Q]+Q H_{n}[Q], \quad n=1,2, \ldots,
$$

с начальным условием

$$
H_{1}[Q]=Q
$$

Для нескольких первых рекуррентных соотношений имеем

$$
\begin{aligned}
& H_{2}=Q_{x}+Q^{2}, \quad H_{3}=Q_{x x}+3 Q Q_{x}+Q^{3}, \\
& H_{4}=Q_{x x x}+4 Q Q_{x x}+3\left(Q_{x}\right)^{2}+6 Q^{2} Q_{x}+Q^{4}, \ldots
\end{aligned}
$$

Различные совокупности рекуррентных соотношений порождают определенные иерархии нелинейных уравнений, линеаризуемых с помощью предложенного метода. Приведем несколько примеров.

Используя соотношения (14) и полагая в качестве условия связи соотношение

$$
V=\sum_{n=1}^{N} C_{n}(x, t) G_{n}[U]
$$

которое соответствует базовому уравнению

$$
\sum_{n=1}^{N} C_{n}(x, t) T^{[n, 0]}=T_{t}
$$

получаем последовательную цепочку уравнений

$$
\frac{\partial}{\partial t} U+\frac{\partial}{\partial x}\left[U \sum_{n=1}^{N} C_{n}(x, t) G_{n}[U]\right]=a \frac{\partial^{2}}{\partial x^{2}} \sum_{n=1}^{N} C_{n}(x, t) G_{n}[U]
$$

при произвольных функциях $C_{n}(x, t)$, которая аналогична высшим уравнениям иерархии КдФ, но интегрируется не с помощью метода обратной задачи рассеяния, а линеаризуется с помощью обобщенной подстановки Коула-Хопфа (11). Первые уравнения этой иерархии, соответствующие $G_{1}, G_{2}, G_{3}, \ldots$ и постоянным коэффициентам $C_{n}$, имеют следующий вид:

$$
\begin{aligned}
& U_{t}+C_{1} \frac{\partial}{\partial x} U=0 \\
& U_{t}+C_{2} \frac{\partial}{\partial x}\left(U^{2}-U_{x}\right)=0 \\
& U_{t}+C_{3} \frac{\partial}{\partial x}\left(3 U U_{x}-U^{3}-U_{x x}\right)=0 \\
& U_{t}+C_{4} \frac{\partial}{\partial x}\left(4 U U_{x x}+3 U_{x}^{2}-6 U^{2} U_{x}+U^{4}-U_{x x x}\right)=0
\end{aligned}
$$


Первым нетривиальным уравнением в этой цепочке при $n=1$ в (14) является уравнение простой волны, затем уравнение Бюргерса, уравнение мКдФ и т.д. Все эти уравнения принадлежат общему классу уравнений, полученных в работе [4].

Эту иерархию можно преобразовать в более сложную иерархию уравнений. Именно, вместо (22) рассмотрим базовое соотношение вида

$$
\sum_{n=1}^{N} C_{n}(x, t) T^{[n, 0]}=T_{t t}
$$

полученное из (22) заменой в правой части первой производной функции $T$ по времени на вторую. Этому базовому соотношению соответствуют связи

$$
V_{t}-V V_{x}+V^{2} U=V F_{N}[U]
$$

где

$$
F_{N}[U]=\left(\sum_{n=1}^{N} C_{n}(x, t) G_{n}[U]\right)^{-1} .
$$

При этом структурное уравнение (12) принимает вид

$$
U_{t}-\frac{\partial^{2}}{\partial x \partial t} \ln V+\frac{\partial}{\partial x} F_{N}[U]=0 .
$$

Аналогичным образом могут быть получены и другие типы иерархий, связанные с рекуррентной цепочкой соотношений (14).

\section{4. УРАВНЕНИЯ НЕЛИНЕЙНОЙ ДВУХПРОВОДНОЙ ЛИНИИ}

Уравнения вида (26) представляют собой нелинейные уравнения типа телеграфного уравнения и встречаются в теории двухпроводных нелинейных линий связи [19], [24], [25]. Уравнения таких линий можно представить в следующем общем виде:

$$
\begin{aligned}
& \frac{\partial i}{\partial \xi}+C \frac{\partial u}{\partial \tau}+J(u)=0 \\
& \frac{\partial u}{\partial \xi}+L \frac{\partial i}{\partial \tau}+I(u)=0
\end{aligned}
$$

или

$$
\frac{\partial}{\partial \tau}\left[C \frac{\partial u}{\partial \tau}\right]-\frac{\partial}{\partial \xi}\left[\frac{1}{L} \frac{\partial u}{\partial \xi}\right]+\frac{\partial}{\partial \tau} J(u)-\frac{\partial}{\partial \xi} I(u)=0,
$$

где $C, L$ - погонные индуктивность и емкость линии, $J(u), I(u)$ - вольт-амперные характеристики нелинейных активных элементов линии. Нетрудно видеть, что каждое из построенных выше уравнений представляет собой модель двухпроводной линии с определенным типом нелинейных свойств.

Наиболее простыми примерами могут служить два уравнения. Первое из них соответствует следующим соотношениям:

$$
\begin{gathered}
T_{x t}+a T_{t}+b T_{x}=0 \\
V_{x}-U V+a V-b=0 \\
\frac{\partial}{\partial t \partial x} \ln V+\frac{\partial}{\partial t}\left[a-\frac{b}{V}\right]+\frac{\partial}{\partial x}[a V-b]=0
\end{gathered}
$$


где $a, b$ - произвольные заданные функции $x, t$. Второе уравнение соответствует следующим соотношениям:

$$
\begin{gathered}
T_{t t}+\gamma T_{x x}+\alpha T_{t}+\beta T_{x}=0, \\
{\left[V_{t}-V V_{x}+V^{2} U\right]+\gamma U+\alpha V-\beta=0,} \\
\frac{\partial}{\partial t}\left[\frac{V_{t}+\gamma_{x} / 2}{\gamma+V^{2}}\right]+\frac{\partial}{\partial x}\left[\frac{\gamma V_{x}-\gamma_{t} / 2}{\gamma+V^{2}}\right]=\frac{\partial}{\partial t}\left[\frac{\alpha V-\beta}{\gamma+V^{2}}\right]+\frac{\partial}{\partial x}\left[\frac{V(\alpha V-\beta)}{\gamma+V^{2}}\right],
\end{gathered}
$$

где $\alpha, \beta, \gamma-$ произвольные функции $x, t$.

В частности, используя подстановку

$$
V=e^{u}, \quad u=u(\xi, \tau)
$$

где $\xi=x+t, \tau=x-t$, и, полагая для простоты $\alpha=\beta=$ const, приведем уравнение (30) к виду (27):

$$
u_{\xi}+i_{\tau}-2 \alpha \operatorname{sh} u=0, \quad u_{\tau}+i_{\xi}-2 \alpha \operatorname{ch} u=0
$$

или (29) при $C=L=1$ :

$$
u_{\xi \xi}-u_{\tau \tau}+2 \alpha \frac{\partial}{\partial \xi} \operatorname{sh} u+2 \alpha \frac{\partial}{\partial \tau} \operatorname{ch} u=0
$$

Характеристики нелинейных элементов в такой модели имеют простой вид $J(u)=$ $-2 \alpha \operatorname{ch} u, I(u)=2 \alpha \operatorname{sh} u$ и реализуются в линиях с распределенными $(\mathrm{p}-\mathrm{n})$-переходами и биологических мембранах [19], [24], [25].

Динамика напряжений в линиях такого типа описывается общим решением линейного телеграфного уравнения, которое можно представить в виде интеграла Фурье-Лапласа

$$
T(\xi, \tau)=\int_{C} A(k) e^{i k \xi-i \omega(k) \tau} d k
$$

где $\omega(k)= \pm \sqrt{k^{2}-2 \alpha i k}, A(k)$ - произвольная комплексная функция и интеграл берется по некоторому контуру $C$ в комплексной плоскости параметра $k$. Соответствующее решение для $u=\ln V$ может быть записано в виде

$$
u=\ln V=\ln \left\{\frac{\int_{C} A(k)(\omega(k)+k) \exp [i k \xi-i \omega(k) \tau] d k}{\int_{C} A(k)(\omega(k)-k) \exp [i k \xi-i \omega(k) \tau] d k}\right\}
$$

Асимптотические свойства решений такого типа могут быть установлены методом перевала [2].

Отметим, что уравнение (31) и все уравнения типа (26) и аналогичных цепочек могут служить моделями двухпроводных линий с нелинейностью обобщенного вида.

3 Теоретическая и математическая физика, т. 158, № 1, 2009 г. 


\section{5. УРАВНЕНИЯ ГИДРОДИНАМИЧЕСКОГО ТИПА}

Развитый подход имеет еще одну модификацию, позволяющую находить уравнения, которые не линеаризуются, но с помощью тех же подстановок (11) сводятся к некоторому интегрируемому нелинейному дифференциальному уравнению. Систематизацию таких случаев провести сложно. Поэтому мы рассмотрим несколько отдельных примеров, которые могут продемонстрировать некоторые возможности данного подхода и имеют важное прикладное значение.

ПримеР 1. Рассмотрим базовое соотношение

$$
\frac{T_{x x}}{T_{t}}+p \frac{T_{x t}}{T_{x}}=0,
$$

где $p=p(t)$ - произвольная функция $t$. Это уравнение эквивалентно связи следующего вида:

$$
U-p V\left(V_{x}-U V\right)=0 .
$$

Отсюда получаем

$$
U=\frac{p V V_{x}}{1+p V^{2}}
$$

Подставляя это выражение в (12), получаем линеаризуемое уравнение

$$
\frac{\partial}{\partial t}\left[\frac{p V V_{x}}{1+p V^{2}}\right]+\frac{\partial}{\partial x}\left[\frac{V_{x}}{1+p V^{2}}\right]=0,
$$

которое допускает однократное интегрирование по $x$. В результате получаем

$$
\frac{\partial}{\partial t}\left(p V^{2}\right)+V_{x}=C_{0}(t)\left(1+p V^{2}\right),
$$

где $C_{0}(t)$ - постоянная интегрирования, параметрически зависящая от $t$. Это уравнение аналогично уравнению "простых" волн (см., например, [2], [26], [27] и библиографию в них).

Уравнение (32) приводится к виду

$$
\frac{\partial}{\partial x}\left[\left(T_{x}\right)^{2}+p\left(T_{t}\right)^{2}\right]=0,
$$

или

$$
\left[\left(T_{x}\right)^{2}+p\left(T_{t}\right)^{2}\right]=C_{1}(t) .
$$

Это уравнение следует разрешить относительно одной из производных

$$
T_{x}= \pm \sqrt{C_{1}-p\left(T_{t}\right)^{2}} .
$$

Положим $C_{1}=$ const и введем новую переменную $d \tau=(p(t))^{-1 / 2} d t$. Далее, вводя обозначение $\zeta=T_{\tau}$ и дифференцируя это соотношение по $\tau$, приходим к уравнению обрушивающихся волн

$$
\zeta_{x} \mp \frac{\zeta}{\sqrt{C_{1}-\zeta^{2}}} \zeta_{\tau}=0,
$$

решение которого сводится к решению неявного алгебраического уравнения

$$
F\left(\zeta, \tau \sqrt{C_{1}-\zeta^{2}} \mp x \zeta\right)=0,
$$

где $F(\zeta, \xi)$ - произвольная дифференцируемая функция двух аргументов. 
Пример 2. Пример, рассмотренный в работе [22], дает базовое уравнение для $T$ следующего вида:

$$
T_{x x} T_{t t}-\left(T_{x t}\right)^{2}=0 .
$$

Этому уравнению соответствует связь

$$
U\left(V_{t}-V V_{x}+V^{2} U\right)=\left(V_{x}-V U\right)^{2} .
$$

Преобразуя последнее соотношение и объединяя его с (12), получаем систему уравнений гидродинамического типа:

$$
\begin{aligned}
& U\left(V_{t}+V V_{x}\right)=\left(V_{x}\right)^{2}, \\
& U_{t}+\frac{\partial}{\partial x}(U V)=V_{x x} .
\end{aligned}
$$

Умножая второе уравнение (36) на $V$ и складывая его с первым, получаем следующую эквивалентную систему:

$$
\begin{gathered}
\frac{\partial}{\partial t}(U V)+\frac{\partial}{\partial x}\left(U V^{2}\right)=\frac{\partial^{2}}{\partial x^{2}} V^{2} \\
U_{t}+\frac{\partial}{\partial x}(U V)=V_{x x} .
\end{gathered}
$$

Положим

$$
\rho=U, \quad u=V-U^{-1} V_{x}, \quad p=V_{t}+V V_{x}-U^{-1}\left(V_{x}\right)^{2}=0 .
$$

Тогда система (37) приводится к системе уравнений одномерного течения сжимаемого газа

$$
\begin{aligned}
\frac{\partial}{\partial t}(\rho u)+\frac{\partial}{\partial x}\left(\rho u^{2}\right) & =-\frac{\partial}{\partial x} p \\
\rho_{t}+\frac{\partial}{\partial x}(\rho u) & =0
\end{aligned}
$$

где $\rho$ - плотность сжимаемого газа, $u$ - скорость течения газа, $p$ - давление. В силу уравнения (36) давление в этом случае обращается в нуль. Полученные уравнения и их решения соответствуют баротропному течению сжимаемой жидкости.

Решение уравнения (35) будем искать в виде

$$
T_{t}=S\left(T_{x}\right)
$$

где $S(z)$ - произвольная дифференцируемая функция своего аргумента. В результате замены $T_{x}=q$ последнее уравнение приводится к уравнению

$$
q_{t}=S^{\prime}(q) q_{x}
$$

решение которого является решением алгебраического уравнения (см. [2], [27])

$$
F\left(q, x-S^{\prime}(q) t\right)=0
$$


где $F(\xi, \tau)$ - произвольная дифференцируемая функция. После построения решения для $T$ функции $V$ и $U$ находятся с помощью подстановок

$$
V=-\frac{T_{t}}{T_{x}}, \quad \rho=U=-\frac{T_{x x}}{T_{x}}, \quad u=-\frac{T_{x t}}{T_{x x}} .
$$

Учитывая соотношения (38), находим плотность потока, его давление и скорость. Все решения уравнений (39) определяются двумя дифференцируемыми функциями $S$ и $F$.

Нетрудно видеть, что базовое соотношение можно дополнить его квадратичными слагаемыми по производным $T$ таким образом, чтобы получались интегрируемые по $T$ уравнения. Если ввести скобки

$$
\{G, H\}=G_{x} H_{t}-G_{t} H_{x},
$$

то в самом общем виде базовое соотношение, приводящее к системе (39) с ненулевым давлением, можно представить в следующем виде:

$$
\begin{aligned}
p & =\frac{1}{U\left(T_{x}\right)^{2}}\left\{T_{x}, \Phi\right\}-\int \frac{1}{\left(T_{x}\right)^{2}}\left\{T_{x}, \Phi\right\} d x= \\
& =-\frac{1}{U T_{x}}\left[U \Phi_{t}-Q \Phi_{x}\right]+\int \frac{1}{T_{x}}\left[U \Phi_{t}-Q \Phi_{x}\right] d x
\end{aligned}
$$

где $\Phi=\Phi(x, t)$ - произвольная функция $x, t$, при этом

$$
U\left(V_{t}+V V_{x}\right)-\left(V_{x}\right)^{2}=\left\{T_{x}, \Phi\right\} .
$$

Давление в уравнении (39) принимает вид

$$
p=U^{-1}\left\{T_{x}, \Phi\right\}-\int\left\{T_{x}, \Phi\right\} d x,
$$

а интегралом базового соотношения (42) будет уравнение

$$
T_{t}=S\left(T_{x}\right)+\Phi(x, t) .
$$

Функцию $\Phi$ определим следующим образом:

$$
\Phi=\alpha T+\nu T_{x x}+\gamma T_{x x x} .
$$

В результате уравнение (43) принимает вид

$$
T_{t}=S\left(T_{x}\right)+\alpha T+\nu T_{x x}+\gamma T_{x x x},
$$

который при выборе $S(q)=A q+B q^{2}+c q^{3}$ и подходящем подборе постоянных $\alpha$, $\nu, \gamma, A, B, C$ приводит к различным вариантам интегрируемых уравнений для $T$. В частности, при $\gamma=0$ первое уравнение в (39) принимает вид

$$
\frac{\partial}{\partial t}(\rho u)+\frac{\partial}{\partial x}\left(\rho u^{2}\right)-\frac{\partial}{\partial x}\left(\nu \rho \frac{\partial}{\partial x} u\right)=\left(\alpha-\nu \rho^{2}\right) u_{x} .
$$

Уравнение (44) представляет собой одномерное уравнение Навье-Стокса для сжимаемой жидкости с кинематической вязкостью $\nu$, функция в правой части уравнения может рассматриваться как градиент давления в среде. Более общий результат относительно уравнений сжимаемой жидкости изложен в работе [22]. 


\section{6. ЛИНЕАРИЗУЕМЫЕ МАТРИЧНЫЕ УРАВНЕНИЯ}

В случае матриц размерности $M>1$ линеаризуемые с помощью обобщенных подстановок Коула-Хопфа уравнения представляют собой системы нелинейных уравнений. Таких систем гораздо больше, чем в скалярном случае, поэтому приведем лишь несколько простых примеров.

ПримеР 3 . В случае связи $\widehat{Q}=\widehat{C} \widehat{U}$, где $\widehat{C}$ - некоторая постоянная матрица, базовым интегрирующим уравнением является уравнение вида

$$
\widehat{T}_{x t}=\widehat{C} \widehat{T}_{x x} .
$$

Структурное уравнение приобретает в этом случае следующий вид:

$$
\widehat{U}_{t}+[\widehat{C}, \widehat{U}] \widehat{U}-\widehat{C} \widehat{U}_{x}=0 .
$$

Уравнения этого типа имеют квадратичную нелинейность, аналогичную нелинейности уравнений генерации второй гармоники и взаимодействия $n$-волн [16], которые встречаются в нелинейной оптике и акустике. Для иллюстрации структуры получаемых уравнений рассмотрим случай матриц размерности $M=2$. Введем следующие обозначения:

$$
\begin{gathered}
\widehat{U}=\left(\begin{array}{ll}
a(x, t) & u(x, t) \\
v(x, t) & b(x, t)
\end{array}\right), \quad \widehat{C}=\left(\begin{array}{cc}
0 & \lambda \\
-\lambda & 0
\end{array}\right), \\
A(x, t)=a(x, t)+i v(x, t), \quad B(x, t)=b(x, t)+i u(x, t) .
\end{gathered}
$$

В этом случае система (45) будет иметь вид системы уравнений для комплексных амплитуд $A$ и $B$ двух связанных волноводов с квадратичной нелинейностью без дисперсии и с диссипацией:

$$
\begin{aligned}
& A_{t}+i \lambda A_{x}+i \lambda A^{*} B^{*}-i \lambda|A|^{2}=0, \\
& B_{t}-i \lambda B_{x}-i \lambda B^{*} A^{*}+i \lambda|B|^{2}=0 .
\end{aligned}
$$

ПримеР 4. Следующим по сложности матричным нелинейным уравнением, которое линеаризуется с помощью данного подхода, является матричное уравнение, соответствующее связи $\widehat{V}=\widehat{C} \widehat{U}$, где $\widehat{C}$ - также некоторая постоянная матрица. Базовым уравнением, к которому оно приводится, будет матричное уравнение следующего вида:

$$
\widehat{T}_{x x}+\widehat{C} \widehat{T}_{t}=0,
$$

а само уравнение принимает следующий вид:

$$
\widehat{C}^{-1}\left(\widehat{U}_{t}-[\widehat{C}, \widehat{U}] \widehat{U}^{2}\right)+2 \widehat{U}_{x} \widehat{U}+\frac{\partial}{\partial x}\left(\widehat{U}^{2}\right)-\widehat{U}_{x x}=0 .
$$

Система уравнений для элементов матрицы $\widehat{U}$ уже имеет кубическую нелинейность, аналогичную нелинейному уравнению Шредингера. Если матрицы $\widehat{U}$ и $\widehat{C}$ выбрать так же, как в предыдущем примере (46), система (47) будет иметь вид системы уравнений для комплексных амплитуд $A$ и $B$ уже с кубической нелинейностью:

$$
\begin{aligned}
& A_{t}+i \lambda A_{x x}-i \lambda A B_{x}^{*}+i \lambda M_{x}+i \lambda \frac{H}{2}=0, \\
& B_{t}-i \lambda B_{x x}+i \lambda B A_{x}^{*}+i \lambda N_{x}+i \lambda \frac{G}{2}=0,
\end{aligned}
$$


где

$$
\begin{gathered}
M=A^{*} B^{*}-\frac{A^{2}}{2}-|A|^{2}, \quad N=A^{*} B^{*}-\frac{B^{2}}{2}-|B|^{2}, \\
H=A\left[|A|^{2}+|B|^{2}-B A\right]+A^{*}\left[|A|^{2}+|B|^{2}+\left(A+A^{*}\right)\left(B-B^{*}\right)-B A^{*}\right] \\
G=B\left[|A|^{2}-|B|^{2}+B A\right]-B^{*}\left[|A|^{2}-|B|^{2}+\left(A-A^{*}\right)\left(B+B^{*}\right)-B A^{*}\right] .
\end{gathered}
$$

Еще одним полезным примером является простая связь $V=C\left(U_{x}-U^{2}\right)$, которая приводит к матричному обобщению уравнений КдФ и мКдФ, но в силу его громоздкости мы приводить ее не будем. Это уравнение линеаризуется к матричному уравнению

$$
\widehat{T}_{x x x}=\widehat{C} \widehat{T}_{t} .
$$

Следует отметить, что в матричном случае существуют иерархии уравнений, которые строятся по аналогии со скалярным случаем.

\section{7. ЗАКЛЮЧЕНИЕ}

Как было показано, рассмотренный в работе подход является универсальным способом для построения уравнений, которые линеаризуются с помощью подстановок типа Коула-Хопфа. Фактически метод перечисляет все такие уравнения в размерности $1+1$. На конкретных примерах показано, что метод имеет приложения в различных разделах теоретической и математической физики. Сам метод основывается на достаточно общих аналитических свойствах функций общего вида, в том числе и матричных. По всей видимости, он может иметь приложения в более широкой области математической физики, чем простое генерирование линеаризуемых нелинейных уравнений. Однако в таком виде рассмотренные примеры дают ценную информацию о классах полностью интегрируемых нелинейных уравнений, но не с помощью МО3, а с помощью метода линеаризации. Можно, однако, высказать гипотезу, что рассмотренный в данной работе подход тесным образом связан с МО3, поскольку структурные уравнения (6) имеют вид уравнений, используемых в представлении ЛЗШ, интегрируемых с помощью МОЗ. Отличие этих подходов состоит в различных видах ограничений на функции $\widehat{T}$. Для данного подхода это дополнительное уравнение для $\widehat{T}$ общего вида (10), а для МОЗ - это дополнительные условия на спектральные свойства функции $\widehat{T}$ и соответствующих базовым соотношениям линейные операторы. Однако этот вопрос требует отдельного анализа.

\section{Список литературы}

[1] J. M. Burgers, The Nonlinear Diffusion Equation. Asymptotic Solutions and Statistical Problems, Reidel, Dordrecht, Holland, 1974.

[2] Дж. Уизем, Линейные и нелинейные волны, Мир, М., 1978.

[3] C. S. Gardner, J. M. Green, M.D. Kruskal, R. M. Miura, Phys. Rev. Lett., 19:19 (1967), 1095-1097.

[4] С. И. Свинолупов, ТМФ, 65:2 (1985), 303-307.

[5] Н. Х. Ибрагимов, Группь преобразований в математической физике, Наука, М., 1983.

[6] А. В. Михайлов, А. Б. Шабат, Р. И. Ямилов, УМН, 42:4(256) (1987), 3-53.

[7] В. В. Соколов, УМH, 43:5(263) (1988), 133-163.

[8] С. И. Свинолупов, В. В. Соколов, УМН, 47:3(285) (1992), 115-146. 
[9] С. И. Свинолупов, В. В. Соколов, ТМФ, 100:2 (1994), 214-218.

[10] В. Э. Адлер, А. Б. Шабат, Р. И. Ямилов, ТМФ, 125:3 (2000), 355-424.

[11] С. Я. Старцев, ТМФ, 116:3 (1999), 336-348.

[12] С. Я. Старцев, ТМФ, 127:1 (2001), 63-74.

[13] F. Calogero, "Why are certain nonlinear PDEs both widely applicable and integrable?", What is Integrability?, Springer Ser. Nonlinear Dynam., ed. V.E. Zhakharov, Springer, Berlin, 1991, 1-62.

[14] A. I. Zenchuk, P. M. Santini, On the remarkable relations among PDEs integrable by the inverse spectral transform method, by the method of characteristics and by the Hopf-Cole transformation, arXiv: 0801.3945.

[15] P. M. Santini, Inverse Problems, 8:2 (1992), 285-301.

[16] В.Е. Захаров, С.В. Манаков, С. П. Новиков, Л. П. Питаевский, Теория солитонов: Метод обратной задачи, Наука, М., 1980.

[17] Р. Додд, Дж. Эйлбек, Дж. Гиббон, Х. Моррио, Солитоны и нелинейные волновые уравнения, Мир, М., 1988.

[18] В. М. Журавлев, ЖЖЭТФ, 110:6 (1996), 2243-2262.

[19] В. М. Журавлев, Нелинейные волны в многокомпонентных системах с дисперсией и диффузией, Изд. УлГУ, Ульяновск, 2002.

[20] В. М. ЖКуравлев, А. В. Никитин, Третъи Курдюмовские чтения "Синергетика в естественных науках", Материалы межд. междисципл. науч. конф. (Тверь, 19-22 апреля 2007), Тверской гос. ун-т, Тверь, 2007, 60-61.

[21] В. М. Журавлев, А. В. Никитин, Нелинейный мир, 5:9 (2007), 603-611.

[22] В. М. Журавлев, Д. А. Зиновьев, Писъма в ЖЭЭФ, 87:5 (2008), 314-318.

[23] Б. А. Урюков, Теплофизика и аэромеханика, 6:3 (1999), 421-424.

[24] Э. Скотт, Волны в активных и нелинейных средах в приложении к электронике, Советское радио, М., 1977.

[25] В. М. Журавлев, ЖЭТФ, 129:3 (2006), 587-604; Писъма в ЖЭТФ, 75:1 (2002), 11-16.

[26] Л. Д. Ландау, Е. М. Лифшиц, Механика сплошных сред, Гостехиздат, М., 1953.

[27] О. В. Руденко, С. И. Солуян, Теоретические основы нелинейной акустики, Наука, М., 1975.

Поступила в редакцию 11.09.2007, после доработки 25.06.2008 